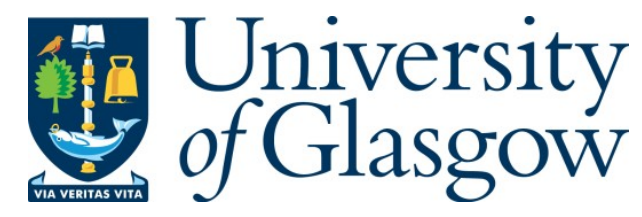

B aker, A ndrew, and Richter, B irgit (2011) Galois theory and Lubin-Tate cochains on classifying spaces. Central European J ournal of $M$ athematics, 9 (5). pp. 1074-1087. ISSN 1895-1074

Copyright $\odot 2011$ V ersita

A copy can be downloaded for personal non-commercial research or study, without prior permission or charge

Content must not be changed in any way or reproduced in any format or medium without the formal permission of the copyright holder(s)

When referring to this work, full bibliographic details must be given

http://eprints.gla.ac.uk/53678/

Deposited on: 06 M arch 2014

Enlighten - Research publications by members of the University of Glasgow http://eprints.gla.ac.uk 


\title{
GALOIS THEORY AND LUBIN-TATE COCHAINS ON CLASSIFYING SPACES
}

\author{
ANDREW BAKER AND BIRGIT RICHTER
}

\begin{abstract}
We consider brave new cochain extensions $F\left(B G_{+}, R\right) \longrightarrow F\left(E G_{+}, R\right)$, where $R$ is either a Lubin-Tate spectrum $E_{n}$ or the related 2-periodic Morava K-theory $K_{n}$, and $G$ is a finite group. When $R$ is an Eilenberg-Mac Lane spectrum, in some good cases such an extension is a $G$-Galois extension in the sense of John Rognes, but not always faithful. We prove that for $E_{n}$ and $K_{n}$ these extensions are always faithful in the $K_{n}$ local category. However, for a cyclic $p$-group $C_{p^{r}}$, the cochain extension $F\left(B C_{p^{r}}, E_{n}\right) \longrightarrow F\left(E C_{p^{r}}, E_{n}\right)$ is not a Galois extension because it ramifies. As a consequence, it follows that the $E_{n}$-theory Eilenberg-Moore spectral sequence for $G$ and $B G$ does not always converge to its expected target.
\end{abstract}

\section{INTRODUCTION}

In the algebraic Galois theory of commutative rings [5], faithful flatness is a property implied by separability. However, in the topological analogue, the brave new Galois theory of Rognes [16], this is not true. The simplest counterexample, due to Ben Wieland [177], is provided by the $C_{2}$-Galois extension

$$
F\left(B C_{2+}, H \mathbb{F}_{2}\right) \longrightarrow F\left(E C_{2+}, H \mathbb{F}_{2}\right) \sim H \mathbb{F}_{2}
$$

which is not faithful. This example relies on the algebraic fact that

$$
\pi_{*}\left(F\left(B C_{2+}, H \mathbb{F}_{2}\right)\right)=H^{-*}\left(B C_{2} ; \mathbb{F}_{2}\right)
$$

is a polynomial algebra and so has finite global dimension.

In this note we consider this question for a Lubin-Tate spectrum $E_{n}$ and the related Morava $K$-theory $K_{n}$, and show that for any finite group $G$, the extension

$$
E_{n}^{B G}=F\left(B G_{+}, E_{n}\right) \longrightarrow F\left(E G_{+}, E_{n}\right) \sim E_{n}
$$

is faithful as an $E_{n}$-module. We also show that the non-commutative extension

$$
F\left(B G_{+}, K_{n}\right) \longrightarrow F\left(E G_{+}, K_{n}\right) \sim K_{n}
$$

is faithful and $F\left(B G_{+}, K_{n}\right)$ is a faithful $E_{n}$-module. A crucial difference from $F\left(B G_{+}, H \mathbb{F}_{p}\right)$ is that $K_{n}^{*}\left(B G_{+}\right)$is always an Artinian algebra over $\left(K_{n}\right)_{*}$, and so if $K_{n}^{*}\left(B G_{+}\right) \neq K_{n}^{*}$ then it has infinite global dimension by Proposition [2.2.

Our approach to this involves introducing an analogue of the algebraic socle series for a module over an Artinian ring, and we show that this behaves well enough to prove our result.

We show in Section 5 that for a cyclic $p$-group $C_{p^{r}}$, the cochain extension $F\left(B C_{p^{r}+}, E_{n}\right) \longrightarrow$ $F\left(E C_{p^{r}+}, E_{n}\right)$ is ramified and hence it is not a Galois extension. As a consequence it follows that the $E_{n}$-theory Eilenberg-Moore spectral sequence for such groups does not converge to its expected target, whereas work of Tilman Bauer indicates that this is not the case for Morava $K$-theory.

Date: 30/05/2011 version 6: to appear in Central European Journal of Mathematics arxiv:1005.1662 . 2000 Mathematics Subject Classification. Primary 55P43; Secondary 13B05 55N22 55P60.

Key words and phrases. Lubin-Tate spectrum, Morava $K$-theory, classifying spaces of finite groups, Galois extensions.

The second author was supported by the Glasgow Mathematical Journal Learning and Research Support Fund, and she thanks the Mathematics Department of the University of Glasgow for its hospitality. We would like to thank Tilman Bauer, Ken Brown, Jim Davis, Uli Krähmer and Jesper Møller for helpful comments. 
Notation, etc. In discussing purely algebraic notions we will often use boldface symbols $\boldsymbol{A}, \boldsymbol{M}, \ldots$ to denote rings, modules, etc, while for topological objects such as $S$-algebras and their modules we will use italic symbols $A, M, \ldots$, thereby hopefully reducing the possibility of confusion between the two settings. For an associative $S$-algebra $A$, we denote by $\mathscr{D}_{A}$ the derived category of $A$-module spectra defined in [6, chapter III, construction 2.11].

We follow Lam [II, theorem 19.1] in using the phrase local ring to indicate a ring with a unique maximal left ideal (necessarily 2-sided and equal to its Jacobson radical); the quotient of such a ring by its Jacobson radical is a division ring. For non-commutative rings other terminology is often encountered such as scalar local ring.

Brave new Galois extensions. The following definition of a Galois extension is due to John Rognes [16]. Let $A$ be a commutative $S$-algebra and let $B$ be a commutative cofibrant $A$ algebra. Let $G$ be a finite (discrete) group and suppose that there is an action of $G$ on $B$ by commutative $A$-algebra morphisms. Then $B / A$ is a $G$-Galois extension if it satisfies the following two conditions:

- The natural map

$$
A \longrightarrow B^{h G}=F\left(E G_{+}, B\right)^{G}
$$

is a weak equivalence of $A$-algebras.

- There is a natural equivalence of $B$-algebras

$$
\Theta: B \wedge_{A} B \stackrel{\sim}{\rightarrow} F\left(G_{+}, B\right)
$$

induced from the action of $G$ on the right hand factor of $B$.

Furthermore, $B / A$ is a faithful $G$-Galois extension if it also satisfies

- $B$ is faithful as an $A$-module, i.e., for any $A$-module $M, B \wedge_{A} M \sim *$ implies that $M \sim *$. Examples like (ㅁ. ) show that not every Galois extension is faithful.

\section{Recollections on modules over Artinian algebras}

In this section we review some standard algebraic background material; good sources for this are [I, П1].

Let $\boldsymbol{D}$ be a division ring. A ring $\boldsymbol{A}$ equipped with homomorphisms of rings $\eta: \boldsymbol{D} \longrightarrow \boldsymbol{A}$ and $\varepsilon: \boldsymbol{A} \longrightarrow \boldsymbol{D}$ is an augmented $\boldsymbol{D}$-algebra if the following diagram commutes.

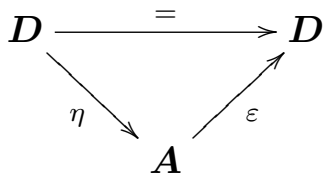

The augmentation $\varepsilon$ splits the unit $\eta$. We will also say that $\boldsymbol{A}$ is an Artinian local $\boldsymbol{D}$-algebra if it is Artinian and local.

If $\boldsymbol{A}$ is an Artinian local augmented $\boldsymbol{D}$-algebra, then the Jacobson radical of $\boldsymbol{A}$ is

$$
\boldsymbol{J}=\operatorname{rad}(\boldsymbol{A})=\operatorname{ker} \varepsilon .
$$

By [II, theorem 4.12], $\boldsymbol{J}$ is nilpotent, say $\boldsymbol{J}^{e}=0$ and $\boldsymbol{J}^{e-1} \neq 0$.

Lemma 2.1. Let $\boldsymbol{A}$ be as above and let $\boldsymbol{M}$ be a left $\boldsymbol{A}$-module. If $\boldsymbol{D} \otimes_{\boldsymbol{A}} \boldsymbol{M}=0$, then $\boldsymbol{M}=0$.

Proof. Comparing the two horizontal exact sequences

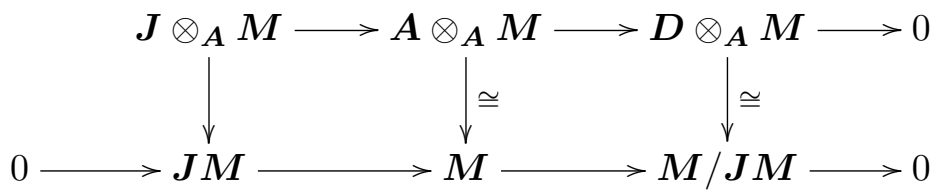

we see that if $\boldsymbol{D} \otimes_{\boldsymbol{A}} \boldsymbol{M}=0$ then

$$
\boldsymbol{M}=\boldsymbol{J} \boldsymbol{M}=\ldots=\boldsymbol{J}^{e} \boldsymbol{M}=0 .
$$


Let $\boldsymbol{M}$ be a left $\boldsymbol{A}$-module. The socle of $\boldsymbol{M}$ is the submodule

$$
\operatorname{soc}^{1} \boldsymbol{M}=\operatorname{soc} \boldsymbol{M}=\{x \in \boldsymbol{M}: \boldsymbol{J} x=0\},
$$

which can also be characterized as the sum of all the simple $\boldsymbol{A}$-submodules of $\boldsymbol{M}$. The socle series of $\boldsymbol{M}$ is the increasing sequence of submodules

$$
0=\operatorname{soc}^{0} \boldsymbol{M} \subseteq \operatorname{soc}^{1} \boldsymbol{M} \subseteq \ldots \subseteq \operatorname{soc}^{k} \boldsymbol{M} \subseteq \operatorname{soc}^{k+1} \boldsymbol{M} \subseteq \ldots \subseteq \boldsymbol{M},
$$

where for each $k$ the following is a pullback square

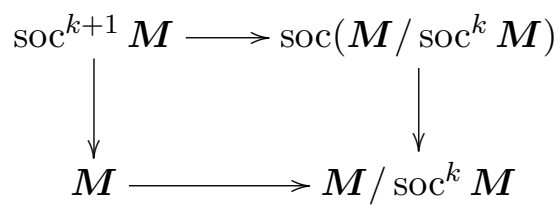

so we have

$$
\operatorname{soc}^{k} \boldsymbol{M}=\left\{x \in \boldsymbol{M}: \boldsymbol{J}^{k} x=0\right\},
$$

and

$$
\operatorname{soc}^{e} M=M .
$$

In fact, for small $k$

$$
\operatorname{soc}^{k} \boldsymbol{M} \subset \operatorname{soc}^{k+1} \boldsymbol{M},
$$

until we reach a value $k=k_{0} \leqslant e$ for which $\operatorname{soc}^{k_{0}} \boldsymbol{M}=\boldsymbol{M}$.

It is also clear that given a homomorphism $\varphi: M \longrightarrow \boldsymbol{M}$ of $\boldsymbol{A}$-modules there are compatible homomorphisms

$$
\operatorname{soc}^{k} \boldsymbol{M} \longrightarrow \operatorname{soc}^{k} \boldsymbol{N} \text {. }
$$

For details on the socle series see [प]], especially Ex. 4.18, and [I, chapter I, section 1].

We end this section with a result that supplies an algebraic backdrop for some of our later work. We give a proof suggested by K. Brown.

Proposition 2.2. Let $\boldsymbol{A}$ be a local left-Artinian ring which is not a division ring. Then

$$
\operatorname{proj} \operatorname{dim}(\boldsymbol{A} / \operatorname{rad}(\boldsymbol{A}))=\mathrm{gl} \operatorname{dim} \boldsymbol{A}=\infty,
$$

where $\boldsymbol{A} / \operatorname{rad}(\boldsymbol{A})$ is the unique simple left $\boldsymbol{A}$-module.

Proof. Since $\boldsymbol{A}$ is local, it has only one simple module and therefore

$$
\operatorname{proj} \operatorname{dim}(\boldsymbol{A} / \operatorname{rad}(\boldsymbol{A}))=\mathrm{gl} \operatorname{dim} \boldsymbol{A} .
$$

Also, since $\boldsymbol{A}$ is Artinian it has a left ideal $\boldsymbol{I}$ isomorphic to $\boldsymbol{A} / \operatorname{rad}(\boldsymbol{A})$. The corresponding exact sequence

$$
0 \rightarrow \boldsymbol{I} \longrightarrow \boldsymbol{A} \longrightarrow \boldsymbol{A} / \boldsymbol{I} \rightarrow 0
$$

cannot split since $\boldsymbol{A}$ is local and therefore it has no non-trivial idempotents.

If

$$
\operatorname{proj} \operatorname{dim}(\boldsymbol{A} / \operatorname{rad}(\boldsymbol{A}))=\mathrm{gl} \operatorname{dim} \boldsymbol{A}<\infty,
$$

then (ए.D) would give

$\operatorname{proj} \operatorname{dim}(\boldsymbol{A} / \operatorname{rad}(\boldsymbol{A}))+1=\operatorname{proj} \operatorname{dim}(\boldsymbol{A} / \boldsymbol{I}) \leqslant \operatorname{gl} \operatorname{dim} \boldsymbol{A}=\operatorname{proj} \operatorname{dim}(\boldsymbol{A} / \operatorname{rad}(\boldsymbol{A}))$,

which is impossible.

Remark 2.3. We end this section by noting that the above discussion works as well if we assume that $\boldsymbol{A}$ is graded, provided this is suitably interpreted. In our work below we are interested in $\mathbb{Z}$-gradings which are also 2-periodic, i.e., for all $n \in \mathbb{Z},(-)_{n+2}=(-)_{n}$. This can be interpreted as a $\mathbb{Z} / 2$-grading. 


\section{SoCle SERIES IN TOPOLOGY}

Let $D$ be an $S$-algebra for which $\pi_{0} D$ is a non-trivial division ring, $\pi_{1} D=0$, and the graded ring $\pi_{*} D=\boldsymbol{D}$ has period two. Suppose that $A$ is an $S$-algebra both under and over $D$, giving the following diagram of morphisms of $S$-algebras.

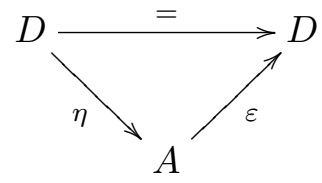

We assume that $\boldsymbol{A}=\pi_{*} A$ is an Artinian local augmented $\boldsymbol{D}$-algebra, so that the augmentation ideal $\operatorname{ker} \varepsilon$ is the Jacobson radical of $\boldsymbol{A}, \operatorname{rad}(\boldsymbol{A})$, and also $\operatorname{rad}(\boldsymbol{A})^{e}=0$ and $\operatorname{rad}(\boldsymbol{A})^{e-1} \neq 0$.

Remark 3.1. Let $M$ be a left $A$-module. Then $\boldsymbol{M}=\pi_{*} M$ is a left $\boldsymbol{A}$-module and its socle soc $\boldsymbol{M}$ is a $\boldsymbol{D}$-module through both the unit $\eta$ and the augmentation $\varepsilon$, and these module structures agree since $\operatorname{rad}(\boldsymbol{A})=\operatorname{ker} \varepsilon$.

Theorem 3.2. There are functors $\operatorname{soc}^{k}: \mathscr{D}_{A} \longrightarrow \mathscr{D}_{A}$ for $0 \leqslant k \leqslant e$ such that

(a) for each $k, \pi_{*}\left(\operatorname{soc}^{k} M\right)=\operatorname{soc}^{k} \boldsymbol{M}$;

(b) there are natural transformations $\operatorname{soc}^{k} M \longrightarrow \operatorname{soc}^{k+1} M$ giving a commutative diagram

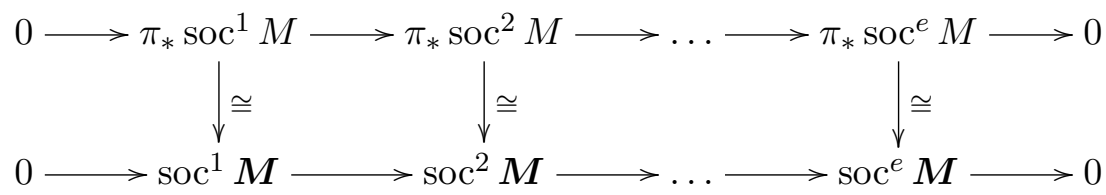

which is natural with respect to morphisms of A-modules.

Proof. As $\boldsymbol{D}$ is a graded division ring, soc $\boldsymbol{M}$ is a $\boldsymbol{D}$-vector space. Since $M$ is a $D$-module via the unit we can find a morphism of $D$-modules

$$
\bigvee_{j} \Sigma^{s(j)} D \longrightarrow M
$$

to realize an algebraic isomorphism

$$
\bigoplus_{j} D_{*-s(j)} \stackrel{\cong}{\longrightarrow} \operatorname{soc} M \subseteq M
$$

Now Remark 3.0 implies that the morphism of (B.2) is actually one of $A$-modules. We set $\operatorname{soc} M=\bigvee_{j} \Sigma^{s(j)} D$.

Now we can repeat this on the cofibre $M / \operatorname{soc} M$ of the map $\operatorname{soc} M \longrightarrow M$, obtaining $\operatorname{soc}(M / \operatorname{soc} M) \longrightarrow M / \operatorname{soc} M$. We then define $\operatorname{soc}^{2} M$ using the right hand pullback square in the diagram

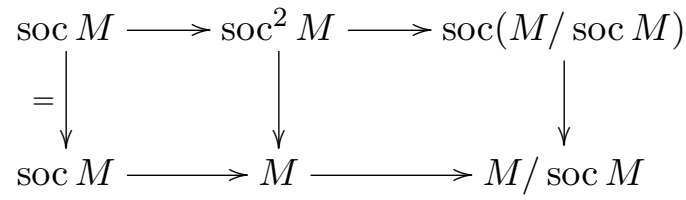

from which we see by a standard diagram chase that $\pi_{*}\left(\operatorname{soc}^{2} M\right) \cong \operatorname{soc}^{2} \boldsymbol{M}$. Continuing in this way we inductively build the socle tower

$$
* \rightarrow \operatorname{soc}^{1} M \longrightarrow \operatorname{soc}^{2} M \longrightarrow \ldots \longrightarrow \operatorname{soc}^{e-1} M \longrightarrow \operatorname{soc}^{e} M=M,
$$

using pullback squares

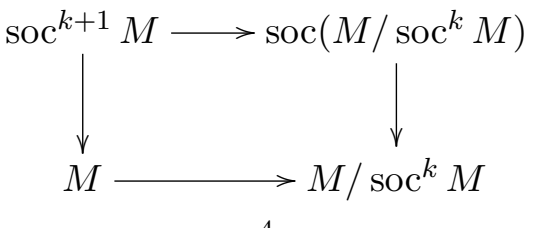


for each $k$. These satisfy

$$
\pi_{*}\left(\operatorname{soc}^{k} M\right)=\operatorname{soc}^{k} M .
$$

An important consequence of this construction is that there is a minimal $k_{0}$ for which $\operatorname{soc}^{k_{0}} M=M$, so since $\operatorname{soc}^{k_{0}-1} \boldsymbol{M} \neq \boldsymbol{M}$, using the fibre sequence

$$
\operatorname{soc}^{k_{0}-1} M \longrightarrow M \longrightarrow M / \operatorname{soc}^{k_{0}-1} M,
$$

we obtain $\pi_{*}\left(M / \operatorname{soc}^{k_{0}-1} M\right) \neq 0$.

Lemma 3.3. The A-module $D$ satisfies $\pi_{*}\left(D \wedge_{A} D\right) \neq 0$.

Proof. There is a diagram of left $D$-modules induced from (B.D)

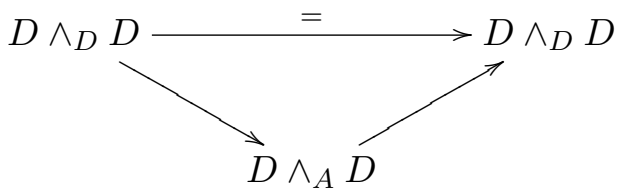

in which $D \wedge_{D} D \cong D$. On applying $\pi_{*}(-)$ we see that $\pi_{*}\left(D \wedge_{A} D\right) \neq 0$.

Theorem 3.4. Let $M$ be an $A$-module for which $\pi_{*} M \neq 0$. Then $\pi_{*}\left(D \wedge_{A} M\right) \neq 0$, i.e., $D$ is a faithful A-module.

Proof. Using the socle series we can find a fibration sequence as in (3.3),

$$
M^{\prime} \longrightarrow M \longrightarrow M^{\prime \prime} \text {, }
$$

where $\boldsymbol{M}^{\prime \prime}=\pi_{*} M^{\prime \prime} \neq 0, J \boldsymbol{M}^{\prime \prime}=0$ and there is a short exact sequence

$$
0 \rightarrow \pi_{*}\left(M^{\prime}\right) \longrightarrow \pi_{*}(M) \longrightarrow \pi_{*}\left(M^{\prime \prime}\right) \rightarrow 0 .
$$

As remarked in the proof of Theorem $\mathbf{3 . 2}, M^{\prime \prime}$ is weakly equivalent to a wedge of copies of suspensions of the $A$-module $D$. So $\pi_{*}\left(M^{\prime \prime}\right)$ is a direct sum of copies of suspensions of $\pi_{*}(D)$, hence by Lemma [3.3, $\pi_{*}\left(M^{\prime \prime}\right) \neq 0$. The fibre sequence (B.4) induces a commutative diagram

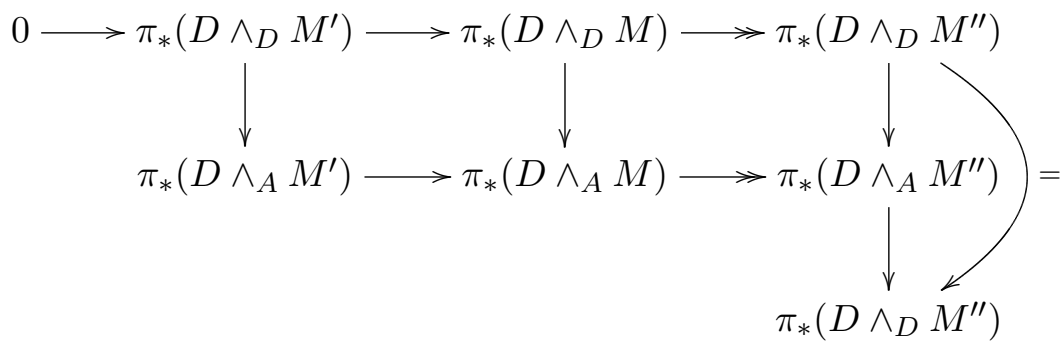

in which a non-zero element $x \in \pi_{*}\left(D \wedge_{D} M^{\prime \prime}\right)$ lifts to $\pi_{*}\left(D \wedge_{D} M\right)$ and so is in the image of composition passing through $\pi_{*}\left(D \wedge_{A} M\right)$. Therefore $\pi_{*}\left(D \wedge_{A} M\right) \neq 0$.

\section{Lubin-TAte COHOMOlOGy of CLASSifying SPACES}

We will denote by $E$ any Lubin-Tate spectrum such as $E_{n}$ or $E_{n}^{\text {nr }}$, and then $K$ will denote the corresponding version of Morava $K$-theory see [Z] for details. The spectrum $E$ is a commutative $S$-algebra, while $K$ is an $E$-algebra in the sense of [6]. The homotopy groups $\pi_{*} E$ and $\pi_{*} K$ are 2-periodic and $\pi_{0} E$ is Noetherian; $\pi_{0} K$ is a field, although $K$ is only homotopy commutative if $p$ is an odd prime, while when $p=2$ it is not even that. Nevertheless, we will view $K$ as a kind of 'topological division ring'.

The following lemma will allows us in certain circumstances to relate modules over $E^{B G}=$ $F\left(B G_{+}, E\right)$ to modules over $K^{B G}=F\left(B G_{+}, K\right)$. 
Lemma 4.1. For any $E^{B G}$-module $M$, there is isomorphism of $K$-modules

$$
K \wedge_{E} B G M \cong\left(K \wedge_{E} E\right) \wedge_{K \wedge_{E} E^{B G}}\left(K \wedge_{E} M\right) .
$$

In particular, there is an isomorphism of $K$-modules

$$
K \wedge_{E^{B G}} E \cong K \wedge_{K^{B G}} K .
$$

Proof. This follows from an obvious generalization of [6, proposition III.3.10]. Since there are isomorphisms of $E$-algebras $K \cong K \wedge_{E} E$ and $K^{B G} \cong K \wedge_{E} E^{B G}$, for any $E^{B G}$-module $M$,

$$
\begin{aligned}
K \wedge_{E} B G & \cong K \wedge_{E}\left(E \wedge_{E} B G\right. \\
& \cong\left(K \wedge_{K} K\right) \wedge_{E}\left(E \wedge_{E} B G M\right) \\
& \cong\left(K \wedge_{E} E\right) \wedge_{K \wedge_{E} E^{B G}}\left(K \wedge_{E} M\right) .
\end{aligned}
$$

Remark 4.2. By a standard argument making use of the Becker-Gottlieb transfer [4], after $p$-localization, $\Sigma^{\infty} B G_{+}$is a retract of $\Sigma^{\infty} B G_{+}^{\prime}$ where $G^{\prime}$ is any $p$-Sylow subgroup of $G$. In particular, when $p \nmid|G|$ we have

$$
F\left(B G_{+}, E\right) \sim E, \quad F\left(B G_{+}, K\right) \sim K .
$$

Theorem 4.3. Let $G$ be a finite group.

(a) The $K$-cohomology $K^{*}\left(B G_{+}\right)$is a finite dimensional $K^{*}$-vector space and the $E$-cohomology $E^{*}\left(B G_{+}\right)$is a finitely generated $E^{*}$-module.

(b) If $K^{*}\left(B G_{+}\right)$is concentrated in even degrees, then $E^{*}\left(B G_{+}\right)$is a free $E^{*}$-module of finite rank and

$$
K^{*}\left(B G_{+}\right)=K^{*} \otimes_{E^{*}} E^{*}\left(B G_{+}\right)=E^{*}\left(B G_{+}\right) / \mathfrak{m} E^{*}\left(B G_{+}\right) .
$$

(c) $K^{*}\left(B G_{+}\right)$is an augmented Artinian local $K^{*}$-algebra whose maximal ideal is nilpotent. Hence $E^{*}\left(B G_{+}\right)$is an augmented pro-Artinian local $E^{*}$-algebra,

$$
E^{*}\left(B G_{+}\right)=\lim _{r} E^{*}\left(B G_{+}\right) / \mathfrak{m}^{r} E^{*}\left(B G_{+}\right) .
$$

Proof. (a) See [ $[\mathbf{7}, \boldsymbol{\nabla}]$ for example.

(b) See [9, proposition 2.5].

(c) Following Remark 4.2, we can reduce to the case where $G$ is a $p$-group using the transfer associated with a $p$-Sylow subgroup $G^{\prime} \leqslant G$. The case of a cyclic $p$-group $C_{p^{r}}$ is well known and

$$
K^{*}\left(B C_{p^{r}+}\right)=K^{*}[y] /\left(y^{p^{r}}\right) .
$$

The case of a general $p$-group $G$ of order $p^{m}$ follows by induction on $m$ since there is always a normal subgroup $N \triangleleft G$ of index $p$ and this permits an argument with the Serre spectral sequence associated with the fibration

$$
B N \longrightarrow B G \longrightarrow B C_{p}
$$

as used in [13] to calculate $K^{*}\left(B G_{+}\right)$from knowledge of $K^{*}\left(B N_{+}\right)$as input.

It is known that $K^{*}\left(B G_{+}\right)$need not be concentrated in even degrees [10].

We are interested in the $E$-algebras $E^{B G}=F\left(B G_{+}, E\right)$ and $K^{B G}=F\left(B G_{+}, K\right)$, each of which is $K$-local. Of course the diagonal $B G \longrightarrow B G \times B G$ induces the product on each of these, but only $E^{B G}$ is strictly commutative, while $K^{B G}$ is homotopy commutative when $p \neq 2$ and merely associative when $p=2$. At the level of homotopy groups, $E^{*}\left(B G_{+}\right)=\pi_{*}\left(E^{B G}\right)$ and $K^{*}\left(B G_{+}\right)=\pi_{*}\left(K^{B G}\right)$ are both graded commutative.

Now we can apply our earlier results to give

Theorem 4.4. For any finite group $G, E$ and $K$ are faithful $E^{B G}$-modules in the $K$-local category. 
Proof. It suffices to show that $K$ is faithful. By Lemma 4.1 , for any $E^{B G}$-module there is an isomorphism

$$
K \wedge_{E^{B G}} M \cong\left(K \wedge_{E} E\right) \wedge_{K \wedge_{E} E^{B G}}\left(K \wedge_{E} M\right)
$$

The natural morphism of $E$-algebras

$$
K \wedge_{E} F\left(B G_{+}, E\right) \longrightarrow F\left(B G_{+}, K \wedge_{E} E\right)
$$

is a weak equivalence since $K$ is a finite cell $E$-module, so by [6, theorem III.4.2] it is enough to know that

$$
\left(K \wedge_{E} E\right) \wedge_{K^{B G}}\left(K \wedge_{E} M\right) \cong K \wedge_{K^{B G}}\left(K \wedge_{E} M\right) \nsim * .
$$

If $M$ is $K$-local and non-trivial, then $K \wedge_{K^{B G}}\left(K \wedge_{E} M\right) \nsim *$, because we know from Theorem 3.4 that $K$ is faithful as a $K^{B G}$-module.

\section{GALOIS THEORY AND $E^{B G}$}

In this section we will consider extensions of the form

$$
E^{B G}=F\left(B G_{+}, E\right) \longrightarrow F\left(E G_{+}, E\right) \sim E
$$

with $G$ a finite group and consider whether or not they are Galois. Since we know they are faithful, the issue is whether such an extension satisfies the unramified condition that the map

$$
\Theta: F\left(B G_{+}, E\right) \wedge_{E B G} F\left(B G_{+}, E\right) \longrightarrow F\left(G_{+}, E\right)
$$

is weak equivalence, and therefore there is a weak equivalence

$$
E \wedge_{E^{B G}} E \stackrel{\sim}{\longrightarrow} \prod_{G} E
$$

In particular, this condition implies that $\pi_{*}\left(E \wedge_{E^{B G}} E\right)$ is concentrated in even degrees.

We begin by considering the case of cyclic $p$-groups $C_{p^{r}}$.

Theorem 5.1. For each $r \geqslant 1$, the extension

$$
E^{B C_{p^{r}}}=F\left(B C_{p^{r}+}, E\right) \longrightarrow F\left(E C_{p^{r}+}, E\right)
$$

is ramified and hence it is not $C_{p^{r}}$-Galois.

Proof. We recall (see for example [8, lemma 5.1]) that

$$
\left(E^{B C_{p^{r}}}\right)_{*}=E^{*}[[y]] /\left(\left[p^{r}\right] y\right),
$$

where $y \in\left(E^{B C_{p^{r}}}\right)_{0}=E^{0}\left(B C_{p^{r}}\right)$ and the $p$-series $[p] y$ has the form

$$
[p] y \equiv y^{p^{n}} \quad \bmod \mathfrak{m}
$$

so for each $r \geqslant 1$ the $p^{r}$-series is inductively defined by

$$
\begin{aligned}
{\left[p^{r}\right] y=[p]\left(\left[p^{r-1}\right] y\right) } & =p^{r} y+\cdots+y^{p^{r n}}+\cdots \\
& \equiv y^{p^{r n}} \bmod \mathfrak{m} .
\end{aligned}
$$

By the Weierstrass preparation theorem, there is a polynomial

$$
\left\langle p^{r}\right\rangle y=p^{r}+\cdots+y^{p^{r n}-1} \equiv y^{p^{r n}-1} \bmod \mathfrak{m}
$$

for which

$$
\left[p^{r}\right] y=y\left\langle p^{r}\right\rangle y\left(1+y f_{r}(y)\right)
$$

where $f_{r}(y) \in E^{*}[[y]]$. Then we have

$$
\left(E^{B C_{p^{r}}}\right)_{*}=E^{*}[[y]] /\left(y\left\langle p^{r}\right\rangle y\right) .
$$

The $\left(E^{B C_{p^{r}}}\right)_{*}$-module $E_{*}$ admits the periodic minimal free resolution

$$
0 \leftarrow E_{*} \longleftarrow\left(E^{B C_{p^{r}}}\right)_{*} \stackrel{y}{\longleftarrow}\left(E^{B C_{p^{r}}}\right)_{*} \frac{\left\langle p^{r}\right\rangle y}{7}\left(E^{B C_{p^{r}}}\right)_{*} \stackrel{y}{\longleftarrow}\left(E^{B C_{p^{r}}}\right)_{*} \stackrel{\left\langle p^{r}\right\rangle y}{\longleftarrow}\left(E^{B C_{p^{r}}}\right)_{*} \longleftarrow \ldots,
$$


so $\operatorname{Tor}_{*, *}^{\left(E^{B C_{p} r}\right) *}\left(E_{*}, E_{*}\right)$ is the homology of the complex

$$
\begin{aligned}
0 \leftarrow E_{*} \otimes_{\left(E^{B C_{p^{r}}}\right)_{*}}\left(E^{B C_{p^{r}}}\right)_{*} \stackrel{I \otimes y}{\longleftarrow} E_{*} \otimes_{\left(E^{B p^{r}}\right)_{*}}\left(E^{B C_{p^{r}}}\right)_{*} \stackrel{I \otimes\left\langle p^{r}\right\rangle y}{\longleftarrow} E_{*} \otimes_{\left(E^{B C_{p^{r}}}\right)_{*}}\left(E^{B C_{p^{r}}}\right)_{*} \\
\stackrel{I \otimes y}{\longleftarrow} E_{*} \otimes_{\left(E^{B C_{p^{r}}}\right)_{*}}\left(E^{B C_{p^{r}}}\right)_{*} \stackrel{I \otimes\left\langle p^{r}\right\rangle y}{\longleftarrow} E_{*} \otimes_{\left(E^{B C_{p} r}\right)_{*}}\left(E^{B C_{p^{r}}}\right)_{*} \longleftarrow \ldots,
\end{aligned}
$$

which is equivalent to

$$
0 \leftarrow E_{*} \stackrel{0}{\longleftarrow} E_{*} \stackrel{p^{r}}{\longleftarrow} E_{*} \stackrel{0}{\longleftarrow} E_{*} \stackrel{p^{r}}{\longleftarrow} E_{*} \longleftarrow \ldots
$$

Since $E_{*}$ is torsion-free, for $s \geqslant 0$ this gives

$$
\operatorname{Tor}_{s, *}^{\left(E^{B C_{p}}\right)_{*}}\left(E_{*}, E_{*}\right)=\left\{\begin{array}{cl}
E_{*} & \text { if } s=0, \\
E_{*} / p^{r} E_{*} & \text { if } s \text { is odd, } \\
0 & \text { otherwise. }
\end{array}\right.
$$

Thus in the Künneth spectral sequence

$$
\mathrm{E}_{s, t}^{2}=\operatorname{Tor}_{s, t}^{\left(E^{B C_{p^{r}}}\right)_{*}}\left(E_{*}, E_{*}\right) \Longrightarrow \pi_{s+t}\left(E \wedge_{E^{B C_{p^{r}}}} E\right)
$$

there can be no non-trivial differentials since for degree reasons the only possibilities involve $E_{*}$-module homomorphisms of the form

$$
d^{2 k-1}: \mathrm{E}_{2 k-1, t}^{2}=E_{t} / p^{r} E_{t} \longrightarrow \mathrm{E}_{0, t+2 k-2}^{2}=E_{t+2 k-2},
$$

with torsion-free target. This shows that the odd degree terms in $\pi_{*}\left(E \wedge_{E^{B C_{p}}} E\right)$ are not zero, contradicting the unramified condition 5.0 for a Galois extension.

Remark 5.2. If we work rationally, then the Künneth spectral sequence

$$
\mathrm{E}_{s, t}^{2}\left(C_{p^{r}} ; \mathbb{Q}\right)=\operatorname{Tor}_{s, t}^{\left(\left(E^{B C_{p^{r}}}\right) \mathbb{Q}\right)_{*}}\left(E_{*} \mathbb{Q}, E_{*} \mathbb{Q}\right) \Longrightarrow \pi_{s+t}\left(E \mathbb{Q} \wedge_{\left(E^{B C_{p^{r}}}\right) \mathbb{Q}} E \mathbb{Q}\right)
$$

has $\mathrm{E}_{s, *}^{2}\left(C_{p}^{r} ; \mathbb{Q}\right)=0$ except when $s=0$, giving

$$
\pi_{*}\left(E \mathbb{Q} \wedge\left(_{\left(E^{B C^{r}}\right) \mathbb{Q}} E \mathbb{Q}\right)=E_{*} \mathbb{Q} \otimes_{\left(E^{B C_{p} r}\right) * \mathbb{Q}} E_{*} \mathbb{Q} .\right.
$$

This shows that higher filtration terms in the Künneth spectral sequence 5.5 contribute $p$ torsion.

Now we extend Theorem 5.0 to arbitrary $p$-groups.

Theorem 5.3. Let $G$ be a non-trivial p-group. Then the extension

$$
F\left(B G_{+}, E\right) \longrightarrow F\left(E G_{+}, E\right)
$$

is not $G$-Galois. More precisely, this extension is ramified:

$$
F\left(E G_{+}, E\right) \wedge_{F\left(B G_{+}, E\right)} F\left(E G_{+}, E\right) \nsim \prod_{G} F\left(E G_{+}, E\right) .
$$

Proof. Choose a non-trivial epimorphism $G \longrightarrow C_{p}$; then for some $k \geqslant 1$ there is a factorization

$$
C_{p^{k}} \rightleftharpoons G \stackrel{\longrightarrow}{\longrightarrow} C_{p}
$$

inducing morphisms between the associated Künneth spectral sequences

$$
\mathrm{E}_{* *}^{r}\left(C_{p}\right) \longrightarrow \mathrm{E}_{* *}^{r}(G) \longrightarrow \mathrm{E}_{* *}^{r}\left(C_{p^{k}}\right) .
$$

As we saw in the proof of Theorem 5.1 , the two outer spectral sequences have trivial differentials. We will analyze the composite morphism $\mathrm{E}_{* *}^{2}\left(C_{p}\right) \longrightarrow \mathrm{E}_{* *}^{2}\left(C_{p^{k}}\right)$.

On choosing generators appropriately, the canonical epimorphism $C_{p^{k}} \longrightarrow C_{p}$ induces the $E_{*}$-algebra monomorphism

$$
\left(E^{B C_{p}}\right)_{*}=E_{*}[[y]] /([p] y) \longrightarrow\left(E^{B C_{p^{k}}}\right)_{*}=E_{*}[[y]] /\left(\left[p^{k}\right] y\right) ; \quad y \mapsto\left[p^{k-1}\right] y,
$$


hence the induced map between the two resolutions of the form (5.2) is

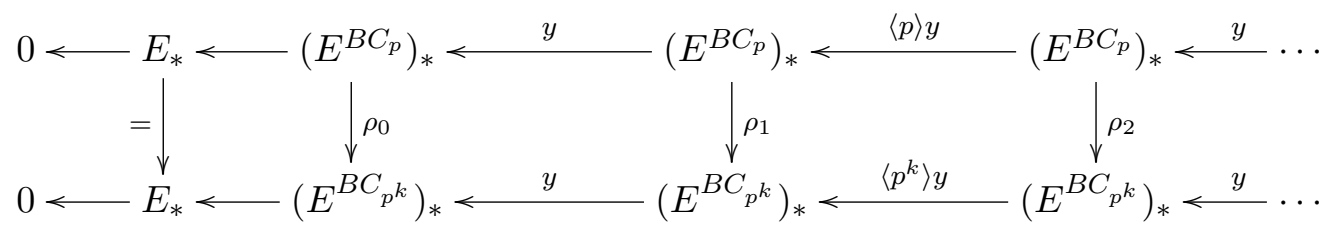

where the vertical maps are given by

$$
\rho_{2 s}: g(y) \mapsto g\left(\left[p^{k-1}\right] y\right), \quad \rho_{2 s-1}: h(y) \mapsto h\left(\left[p^{k-1}\right] y\right)\left\langle p^{k-1}\right\rangle y .
$$

Applying $E_{*} \otimes_{\left(E^{\left.B C_{p^{r}}\right)_{*}}\right.}(-)$ to the first and second rows with $r=1$ and $k$ respectively, we obtain a map of chain complexes

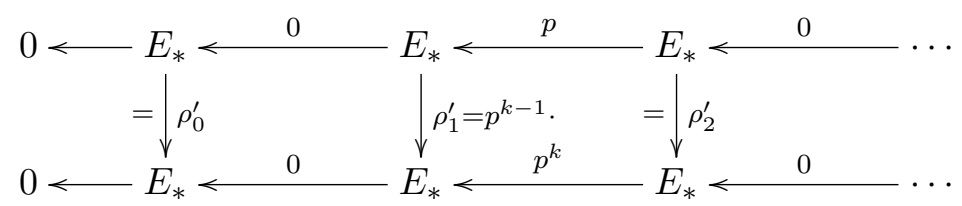

where

$$
\rho_{2 s}^{\prime}=\mathrm{id}, \quad \rho_{2 s-1}^{\prime}=p^{k-1} .
$$

Applying this to the odd degree terms given in (5.4) we see that the induced map

$$
E_{*} / p E_{*} \stackrel{p^{k-1} \cdot}{\longrightarrow} E_{*} / p^{k} E_{*}
$$

is always a monomorphism. Therefore in (5.7), the first of the induced morphisms

$$
\mathrm{E}_{* *}^{2}\left(C_{p}\right) \longrightarrow \mathrm{E}_{* *}^{r}(G) \longrightarrow \mathrm{E}_{* *}^{r}\left(C_{p^{k}}\right)
$$

is a monomorphism. There can be no higher differentials killing elements in its image because they map to non-trivial elements of $\mathrm{E}_{* *}^{2}\left(C_{p^{k}}\right)$ which survive the right hand spectral sequence. This shows that $\mathrm{E}_{* *}^{\infty}(G)$ contains elements of odd degree, and as in the cyclic group case this is incompatible with the unramified condition.

We can extend this result to the class of $p$-nilpotent groups. A finite group $G$ is $p$-nilpotent if one and hence each $p$-Sylow subgroup $P \leqslant G$ has a normal $p$-complement, i.e., there is a normal subgroup $N \triangleleft G$ with $p \nmid|N|$ and $G=P N=P \ltimes N$. A convenient summary of the properties of such groups can be found in [12, section 7], see also [15].

Corollary 5.4. If $G$ is a p-nilpotent group for which $p$ divides $|G|$, then the extension

$$
F\left(B G_{+}, E\right) \longrightarrow F\left(E G_{+}, E\right)
$$

is ramified and so is not $G$-Galois.

Proof. By a result of Tate [18], $G$ being $p$-nilpotent is equivalent to the restriction homomorphism giving an isomorphism

$$
\operatorname{res}_{P}^{G}: H^{*}\left(B G ; \mathbb{F}_{p}\right) \stackrel{\cong}{\longrightarrow} H^{*}\left(B P ; \mathbb{F}_{p}\right),
$$

and in fact it is sufficient that this holds in degree 1. Comparison of the Serre spectral sequences for $K^{*}\left(B G_{+}\right)$and $K^{*}\left(B P_{+}\right)$shows that

$$
K^{*}\left(B G_{+}\right) \stackrel{\cong}{\longrightarrow} K^{*}\left(B P_{+}\right) .
$$

It now follows that

$$
E^{*}\left(B G_{+}\right) \stackrel{\cong}{\longrightarrow} E^{*}\left(B P_{+}\right) .
$$

and the result can be deduced from Theorem 5.3. 
Remark 5.5. The condition of $G$ being a $p$-nilpotent group should not be confused with the condition that the conjugation action of $G$ on $\mathbb{F}_{p}[G]$ is nilpotent. The latter is used in [16, proposition 5.6.3] to ensure convergence of the Eilenberg-Moore spectral sequence and so to prove that for such groups

$$
F\left(B G_{+}, H \mathbb{F}_{p}\right) \longrightarrow F\left(E G_{+}, H \mathbb{F}_{p}\right)
$$

is a $G$-Galois extension. The example of $G=\Sigma_{3}$, the third symmetric group, for the prime $p=2$ illustrates this. For each of the Sylow 2-subgroups

$$
\{\mathrm{id},(1,2)\},\{\mathrm{id},(1,3)\},\{\mathrm{id},(2,3)\}
$$

has as normal complement

$$
N=\{\mathrm{id},(1,2,3),(1,3,2)\},
$$

therefore $\Sigma_{3}$ is 2-nilpotent. However, the $\Sigma_{3}$-module $\mathbb{F}_{2}\left[\Sigma_{3}\right]$ contains the 2-dimensional nontrivial simple submodule

$$
V=\{x(1,2)+y(1,3)+z(2,3): x+y+z=0\},
$$

so by Jordan-Hölder theory every composition series for $\mathbb{F}_{2}\left[\Sigma_{3}\right]$ must have this as a composition factor. Hence the action of $\Sigma_{3}$ on $\mathbb{F}_{2}\left[\Sigma_{3}\right]$ cannot be nilpotent.

\section{Some observations on the Eilenberg-Moore SPeCtral Sequence}

In [16, section 5.6], it is shown that for a finite $p$-group $G$, the Eilenberg-Moore spectral sequence with

$$
\mathrm{E}_{s, t}^{2}=\operatorname{Tor}_{s, t}^{H^{*}\left(B G_{+} ; \mathbb{F}_{p}\right)}\left(\mathbb{F}_{p}, \mathbb{F}_{p}\right)
$$

converges to $\pi_{*}\left(F\left(G_{+}, H \mathbb{F}_{p}\right)\right)=\pi_{*}\left(\prod_{G} \mathbb{F}_{p}\right)$. By comparing it with the Künneth spectral sequence for $\pi_{*}\left(H \mathbb{F}_{p} \wedge_{F\left(B G_{+}, H \mathbb{F}_{p}\right)} H \mathbb{F}_{p}\right)$, it is also shown that

$$
F\left(B G_{+}, H \mathbb{F}_{p}\right) \longrightarrow F\left(E G_{+}, H \mathbb{F}_{p}\right)
$$

is a $G$-Galois extension.

Let us consider in detail the case $G=C_{p}$ for $p$ an odd prime. The case when $p=2$ is similar. First we write

$$
H^{*}\left(B C_{p}\right)=H^{*}\left(B C_{p_{+}} ; \mathbb{F}_{p}\right)=\mathbb{F}_{p}[y] \otimes \Lambda(z),
$$

where $y \in H^{2}\left(B C_{p}\right)$ and $z \in H^{1}\left(B C_{p}\right)$. Then (6.d) becomes

$$
\mathrm{E}_{* *}^{2}=\Gamma(\sigma z) \otimes \Lambda(\sigma y),
$$

where $\sigma y \in \mathrm{E}_{1,-2}^{2}$ and $\sigma z \in \mathrm{E}_{1,-1}^{2}$ are the suspensions of $y$ and $z$, see [14]. Writing $\gamma_{r}=\gamma_{r}(\sigma z)$. The first non-trivial differential is

$$
d^{p-1} \gamma_{p}=\sigma y
$$

and we have

$$
\mathrm{E}_{* *}^{p}=\mathbb{F}_{p}[\zeta] /\left(\zeta^{p}\right) \otimes \Gamma\left(\gamma_{p^{2}}\right) \otimes \Lambda\left(\gamma_{p} \sigma y\right),
$$

where $\zeta$ represents the class of $\sigma z$. The remaining differentials are determined by the formulae

$$
d^{p^{s}-p^{s-1}-1} \gamma_{p^{s}}=\gamma_{p^{s-1}} \sigma y
$$

in

Finally we have

$$
\mathrm{E}_{* *}^{p^{s}-p^{s-1}-1}=\mathbb{F}_{p}[\zeta] /\left(\zeta^{p}\right) \otimes \Gamma\left(\gamma_{p^{s}}\right) \otimes \Lambda\left(\gamma_{p^{s-1}} \sigma y\right)
$$

$$
\mathrm{E}_{* *}^{\infty}=\mathbb{F}_{p}[\zeta] /\left(\zeta^{p}\right)
$$

which is an avatar of $\prod_{C_{p}} \mathbb{F}_{p}$. These differentials are forced by the known answer and multiplicativity, and are also related to the discussion of [14, section 6]. For Lubin-Tate theory $\left(E^{B C_{p^{r}}}\right)_{*}$ is free over $E_{*}$ and the comparison of the Eilenberg-Moore with the Künneth spectral sequence together with our Theorems 5.0 and 5.3 has the following consequence. 
Proposition 6.1. For the cyclic p-group $C_{p^{r}}$ the E-theory Eilenberg-Moore spectral sequence for $B C_{p^{r}}$ with

$$
{ }^{\mathrm{L}-\mathrm{T}} \mathrm{E}_{s, t}^{2}=\operatorname{Tor}^{\left(E^{B C_{p^{r}}}\right) *}\left(E_{*}, E_{*}\right)
$$

does not converge to $\pi_{*}\left(\prod_{C_{p^{r}}} E\right)$.

Just as in the $H \mathbb{F}_{p}$ case, we can compare the Morava $K$-theory based Eilenberg-Moore spectral sequence with the Künneth spectral sequence. Work of Bauer [3] on the convergence of the Cotor-version of this Eilenberg-Moore spectral sequence shows that the corresponding spectral sequence converges for $G=C_{p}$ and odd primes $p$, and therefore

$$
K \wedge_{K^{B C_{p}}} K \sim \prod_{C_{p}} K
$$

The extension of $S$-algebras $K^{B C_{p}} \longrightarrow K^{E C_{p}}$ can be interpreted as a Galois extension of noncommutative $S$-algebras.

\section{REFERENCES}

[1] J. L. Alperin, Local representation theory, Cambridge Studies in Advanced Mathematics 11, Camb. Univ. Press, Cambridge (1986).

[2] G_ Galois extensions of Lubin-Tate spectra, Homology, Homotopy Appl. 10 (2008), 27-43.

[3] T. Bauer, Convergence of the Eilenberg-Moore spectral sequence for generalized cohomology theories, arXiv:0803.3798.

[4] J. C. Becker \& D. H. Gottlieb, The transfer map and fiber bundles, Topology 14 (1975), 1-12.

[5] S. U. Chase, D. K. Harrison \& A. Rosenberg, Galois theory and Galois cohomology of commutative rings, Mem. Amer. Math. Soc. 52 (1965), 15-33.

[6] A. Elmendorf, I. Kriz, M. Mandell \& J. P. May, Rings, modules, and algebras in stable homotopy theory, Mathematical Surveys and Monographs 47 (1999).

[7] M. J. Hopkins, N. J. Kuhn \& D. C. Ravenel, Morava $K$-theories of classifying spaces and generalized characters for finite groups, Lect. Notes in Math. 1509 (1992), 186-209.

[8] _ Generalized group characters and complex oriented cohomology theories, J. Amer. Math. Soc. 13 (2000), 553-594.

[9] M. Hovey \& N. P. Strickland, Morava $K$-theories and localisation, Mem. Amer. Math. Soc. 139 no. 666 (1999).

[10] I. Kriz \& K. P. Lee, Odd-degree elements in the Morava $K(n)$ cohomology of finite groups, Topology Appl. 103 (2000), 229-241.

[11] T. Y. Lam, A First Course in Noncommutative Rings, 2nd edition, Graduate Texts in Mathematics 131, Springer (2001).

[12] J. Møller, Frobenius categories for finite groups of Lie type, Carski Trakt, Poland (2010), http://www.math.ku.dk/ moller/talks/frobenius.pdf.

[13] D. C. Ravenel, Morava $K$-theories and finite groups, Contemp. Math. 12 (1982), 289-292.

[14] D. C. Ravenel \& W. S. Wilson, Morava K-theories of Eilenberg-MacLane spaces and the Conner-Floyd conjecture, Amer. J. Math. 102 (1980), 691-748.

[15] D. J. S. Robinson, A course in the theory of groups, 2nd edition, Springer-Verlag (1996).

[16] J. Rognes, Galois extensions of structured ring spectra, Mem. Amer. Math. Soc. 192 no. 898 (2008), 1-97.

[17] A Galois extension that is not faithful, http://folk.uio.no/rognes/papers/unfaithful.pdf

[18] J. Tate, Nilpotent quotient groups, Topology 3 suppl. 1 (1964), 109-111.

School of Mathematics \& Statistics, University of Glasgow, Glasgow G12 8QW, Scotland.

E-mail address: a.baker@maths.gla.ac.uk

$U R L$ : http://wWW.maths.gla.ac.uk/ ajb

Fachbereich Mathematik der Universität Hamburg, Bundesstrasse 55, 20146 Hamburg, Germany. E-mail address: birgit.richter@uni-hamburg.de $U R L$ : http://www.math.uni-hamburg.de/home/richter/ 